\title{
On Thermodynamic and Kinetic Stability of Synthetic Multifunctional Rigid-Rod $\beta$-Barrel Pores: Evidence for Supramolecular Catalysis
}

\author{
Svetlana Litvinchuk, ${ }^{\dagger}$ Guillaume Bollot, ${ }^{\dagger}$ Jiri Mareda,${ }^{\dagger}$ Abhigyan Som, ${ }^{\dagger}$ Dawn Ronan,${ }^{\dagger}$ \\ Muhammad Raza Shah, ${ }^{\dagger}$ Philippe Perrottet, ${ }^{\dagger}$ Naomi Sakai ${ }^{\dagger}$ and Stefan Matile ${ }^{\dagger *}$ \\ ${ }^{\dagger}$ Department of Organic Chemistry and ${ }^{\dagger}$ Laboratoire de Spectrométrie de Masse, \\ University of Geneva, Geneva, Switzerland
}

\section{Supporting Information}

General. Reagents for synthesis were purchased from Fluka, amino acid derivatives from Novabiochem, egg yolk phosphatidylcholine (EYPC) from Avanti, HATU from Applied Biosystems, ANTS, DPX, and HPTS from Molecular Probes, CF, AcPTS, PTS, PGA (MW 13600 g/mol), ATP, buffers, and salts from Sigma or Fluka-Aldrich. All reactions were performed under argon atmosphere. Column chromatography was carried out on silica gel 60 (Fluka, 40-63 $\mu \mathrm{m}$ ). Analytical (TLC) and preparative thin layer chromatography (PTLC) were performed in silica gel 60 (Fluka, $0.2 \mathrm{~mm}$ ) and silica gel GF (Analtech, $1000 \mu \mathrm{m}$ ), respectively. Purity of the product was verified by using either Jasco HPLC system (PU-980, UV-970, FP-920) or Agilent 1100 Series. $[\alpha]^{20}$ values were recorded on a Jasco P-1030 Polarimeter, melting points (mp) on a heating table from Reichert (Austria), elemental analysis on a Vario EL instrument from Elementar. IR spectra were recorded on a Perkin Elmer Spectrum One FT-IR spectrometer (ATR, Golden Gate) and are reported in $\mathrm{cm}^{-1}$ with band intensities indicated as s (strong), m (medium), w (weak). ESI-MS was performed on a Finnigan MAT SSQ 7000 instrument. ${ }^{1} \mathrm{H}$ and ${ }^{13} \mathrm{C}$ spectra were recorded (as indicated) on either a Bruker $300 \mathrm{MHz}, 400 \mathrm{MHz}$ or $500 \mathrm{MHz}$ spectrometer and are reported as chemical shifts $(\delta)$ in ppm relative to TMS $(\delta=0)$. Spin multiplicities are reported as a singlet $(\mathrm{s})$, doublet $(\mathrm{d})$, triplet $(\mathrm{t})$, with coupling constants $(J)$ given in $\mathrm{Hz}$, or multiplet $(\mathrm{m})$. ${ }^{1} \mathrm{H}$ and ${ }^{13} \mathrm{C}$ resonances were assigned with the aid of additional information from $2 \mathrm{D}$ NMR spectra [H,H-COSY, DEPT 135, HSQC and HMBC]. UV-Vis spectra were measured on a Varian Cary 1 Bio spectrophotometer. Fluorescence measurements were preformed on either a FluoroMax-2 or a FluoroMax-3, Jobin Yvon-Spex. The Mini-Extruder with a polycarbonate membrane, pore size $100 \mathrm{~nm}$, used for LUV preparation was from Avanti. Planar bilayer conductance was recorded using a bilayer chamber with a Delrin cuvette (BCH-13A, Warner) in a Faraday cage (housemade) on a vibration isolation table (63-500 series, TMC) with a bilayer clamp amplifier (BC-525c, Warner), low-pass filtered with a 8-pole Bessel filter (LPF-8, 
Warner), converted (DigiData 1200, Axon) and analyzed by computer (pClamp 8.0, Axon).

Abbreviations. ANTS: 8-aminonaphthalene-1,3,6-trisulfonate; ATP: Adenosine 5'triphosphate; BOC: $t$-Butoxycarbonyl; DMF: $N, N$-Dimethylformamide; EYPCLUVs: Egg yolk phosphatidylcholine large unilamellar vesicles; DPX: $p$ xylenebis(pyridinum)bromide; Fmoc: 9-Fluorenylmethoxycarbonyl; HATU: $N$ [(dimethylamino)-1H-1,2,3-triazolo[4,5- $b$ ]pyridin-1-ylmethylene]- $N$ -

methylmethanaminium hexafluorophosphate $\mathrm{N}$-oxide; HBTU: $\mathrm{N}$-[(1H-benzotriazol1-yl)(dimethylamino)methylene]- $N$-methylmethanaminium hexafluorophosphate $N$ oxide; His: L-Histidine; HPTS: 8-Hydroxy-1,3,6-pyrenetrisulfonate; AcPTS: 8Acetoxy-1,3,6-pyrenetrisulfonate; Leu: L-Leucine; Lys: L-Lysine; PLGA: Poly-Lglutamic acid (PGA); PTS: Pyrene-1,3,6,8-tetrasulfonate; TFA: Trifluoroacetic acid; Trt: Trityl.

$1^{3}, 2^{3}, 3^{2}, 4^{3}, 5^{2}, 6^{3}, 7^{2}, 8^{3}$-Octakis(Gla-OH)-p-octiphenyl 1a. Compound 1a was prepared from commercial Fast Blue B salt in overall nine steps as described in ref (S1), Supporting Information.

H-Leu-His(Trt)-Leu-NH $\mathbf{N H}_{2}$ (1b). Compound 1b was prepared from commercial amino acids in overall four steps as described in ref (S1), Supporting Information.

Fmoc-Lys(Boc)-Leu-His(Trt)-Leu-NH . General procedure A: HBTU (745 mg, $1.96 \mathrm{mmol})$, Fmoc-Lys(Boc)-OH $(787 \mathrm{mg}, 1.68 \mathrm{mmol})$ and triethylamine $(0.62 \mathrm{ml}$, $8.4 \mathrm{mmol})$ were added to a solution of H-Leu-His(Trt)-Leu- $\mathrm{NH}_{2}(872 \mathrm{mg}, 1.40 \mathrm{mmol})$ in $\mathrm{CH}_{2} \mathrm{Cl}_{2}(10 \mathrm{ml})$ at $0{ }^{\circ} \mathrm{C}$. After stirring overnight in the dark at $\mathrm{rt}$, the reaction mixture was diluted with $\mathrm{CH}_{2} \mathrm{Cl}_{2}$, extracted with aqueous $\mathrm{NaHCO}_{3}$, washed with brine, extracted with $1 \mathrm{M}$ aqueous $\mathrm{KHSO}_{4}$, washed with brine, dried over anhydrous $\mathrm{MgSO}_{4}$, and concentrated in vacuo. Purification of the crude product by column chromatography $\left(\mathrm{CH}_{2} \mathrm{Cl}_{2} / \mathrm{MeOH}=20: 1\right.$, then 10:1) yielded pure Fmoc-Lys(Boc)-LeuHis(Trt)-Leu- $\mathrm{NH}_{2}(937 \mathrm{mg}, 64 \%)$ as a colorless powder. TLC $\left(\mathrm{CH}_{2} \mathrm{Cl}_{2} / \mathrm{MeOH} 10: 1\right)$ : $R_{\mathrm{f}} 0.40 ;[\alpha]^{20}{ }_{\mathrm{D}}=-20.1\left(c=1.0, \mathrm{CH}_{3} \mathrm{OH}\right) ; \mathrm{mp}=104-105{ }^{\circ} \mathrm{C} ; \mathrm{IR}: v 3289(\mathrm{~m}), 2955$ (m), 1652 (s), 1515 (s), 1447 (w), 1387 (w), 1246 (s), 1165 (m), 870 (w), 741 (s), 701 (s); ${ }^{1} \mathrm{H}$ NMR $\left(500 \mathrm{MHz}, \mathrm{CD}_{3} \mathrm{OD}\right): \delta 7.78\left(\mathrm{~d},{ }^{3} J(\mathrm{H}, \mathrm{H})=7.3 \mathrm{~Hz}, 2 \mathrm{H}\right), 7.60\left(\mathrm{~d},{ }^{3} J(\mathrm{H}, \mathrm{H})\right.$ $=6.6 \mathrm{~Hz}, 1 \mathrm{H}), 7.58\left(\mathrm{~d},{ }^{3} J(\mathrm{H}, \mathrm{H})=6.6 \mathrm{~Hz}, 1 \mathrm{H}\right), 7.36-7.26(\mathrm{~m}, 14 \mathrm{H}), 7.08-7.05(\mathrm{~m}$, $6 \mathrm{H}), 6.76(\mathrm{~s}, 1 \mathrm{H}), 4.52\left(\mathrm{dd},{ }^{3} J(\mathrm{H}, \mathrm{H})=5.4 \mathrm{~Hz},{ }^{3} J(\mathrm{H}, \mathrm{H})=7.9 \mathrm{~Hz}, 1 \mathrm{H}\right), 4.40-4.23(\mathrm{~m}$, $4 \mathrm{H}), 4.17\left(\mathrm{t},{ }^{3} J(\mathrm{H}, \mathrm{H})=6.8 \mathrm{~Hz}, 1 \mathrm{H}\right), 4.03\left(\mathrm{dd},{ }^{3} J(\mathrm{H}, \mathrm{H})=5.1 \mathrm{~Hz},{ }^{3} J(\mathrm{H}, \mathrm{H})=8.4 \mathrm{~Hz}\right.$, $1 \mathrm{H}), 3.08\left(\mathrm{dd},{ }^{3} J(\mathrm{H}, \mathrm{H})=5.4 \mathrm{~Hz},{ }^{2} J(\mathrm{H}, \mathrm{H})=12.2 \mathrm{~Hz}, 1 \mathrm{H}\right), 3.01\left(\mathrm{t},{ }^{3} J(\mathrm{H}, \mathrm{H})=6.8 \mathrm{~Hz}\right.$, $2 \mathrm{H}), 2.93\left(\mathrm{dd},{ }^{3} J(\mathrm{H}, \mathrm{H})=7.9 \mathrm{~Hz},{ }^{2} J(\mathrm{H}, \mathrm{H})=12.2 \mathrm{~Hz}, 1 \mathrm{H}\right), 1.76-1.28($ several m, $21 \mathrm{H}), 0.90\left(\mathrm{~d},{ }^{3} J(\mathrm{H}, \mathrm{H})=6.3 \mathrm{~Hz}, 3 \mathrm{H}\right), 0.88\left(\mathrm{~d},{ }^{3} J(\mathrm{H}, \mathrm{H})=6.5 \mathrm{~Hz}, 3 \mathrm{H}\right), 0.85\left(\mathrm{~d},{ }^{3} J(\mathrm{H}, \mathrm{H})\right.$ $=6.3 \mathrm{~Hz}, 3 \mathrm{H}), 0.84\left(\mathrm{~d},{ }^{3} \mathrm{~J}(\mathrm{H}, \mathrm{H})=6.5 \mathrm{~Hz}, 3 \mathrm{H}\right) ;{ }^{13} \mathrm{C} \mathrm{NMR}\left(125 \mathrm{MHz}, \mathrm{CD}_{3} \mathrm{OD}\right): \delta 177.6$ (s), 177.6 (s), 175.5 (s), 174.9 (s), 173.2 (s), 164.8 (s), 145.3 (s), 145.1 (s), 143.6 (s), 142.6 (s), 139.6 (d), 137.8 (s), 130.9 (d), 129.3 (d), 129.3 (d), 128.8 (d), 128.2 (d), 126.2 (d), 121.0 (d), 120.9 (d), 79.8 (s), 76.8 (s), $68.2(\mathrm{t}), 57.2(\mathrm{~d}), 55.5$ (d), 53.9 (d), $53.1(\mathrm{~d}), 48.4(\mathrm{~d}), 41.4(\mathrm{t}), 41.1(\mathrm{t}), 41.1(\mathrm{t}), 32.4(\mathrm{t}), 30.9(\mathrm{t}), 30.6(\mathrm{t}), 28.8(\mathrm{q}), 25.9$ (d), 25.8 (d), 24.2 (t), 23.7 (q), 23.5 (q), 21.9 (q), 21.6 (q); MS (ESI, $\mathrm{CH}_{2} \mathrm{Cl}_{2} / \mathrm{MeOH}$ 
9:1): $m / z(\%) 1073(100)[\mathrm{M}+\mathrm{H}]^{+}, 1095(99)[\mathrm{M}+\mathrm{Na}]^{+}, 2147(20)[2 \mathrm{M}+\mathrm{H}]^{+}, 2169(62)$ $[2 \mathrm{M}+\mathrm{Na}]^{+}$.

H-Lys(Boc)-Leu-His(Trt)-Leu-NH $\mathbf{N H}_{2}$ General procedure B: Fmoc-Lys(Boc)-LeuHis(Trt)-Leu- $\mathrm{NH}_{2}$ (937 mg, $\left.0.90 \mathrm{mmol}\right)$ was dissolved in DMF containing 5\% piperidine. After stirring for $15 \mathrm{~min}$ at $\mathrm{rt}$, the mixture was concentrated in vacuo. Purification of the crude product by column chromatography $\left(\mathrm{CH}_{2} \mathrm{Cl}_{2} / \mathrm{MeOH} 10: 1\right)$ yielded pure H-Lys(Boc)-Leu-His(Trt)-Leu- $\mathrm{NH}_{2}(655 \mathrm{mg}, 86 \%)$ as a colorless powder. TLC $\left(\mathrm{CH}_{2} \mathrm{Cl}_{2} / \mathrm{MeOH} 10: 1\right): R_{\mathrm{f}} 0.05 ;[\alpha]_{\mathrm{D}}^{20}=-15.1\left(c=1.0, \mathrm{CH}_{3} \mathrm{OH}\right) ; \mathrm{mp}=$ $97-98{ }^{\circ} \mathrm{C}$; IR: v 3287 (m), 2957 (m), 1652 (s), 1526 (s), 1447 (w), 1365 (w), 1246 (s), 1251(s), 1166 (m), 1036 (w), 867 (w), 739 (m), 700 (s); ${ }^{1} \mathrm{H}$ NMR (500 MHz, $\left.\mathrm{CD}_{3} \mathrm{OD}\right): \delta 7.38-7.35(\mathrm{~m}, 10 \mathrm{H}), 7.13-7.10(\mathrm{~m}, 6 \mathrm{H}), 6.80(\mathrm{~s}, 1 \mathrm{H}), 4.55\left(\mathrm{dd},{ }^{3} J(\mathrm{H}, \mathrm{H})\right.$ $\left.=5.9 \mathrm{~Hz},{ }^{3} J(\mathrm{H}, \mathrm{H})=7.5 \mathrm{~Hz}, 1 \mathrm{H}\right), 4.35-4.29(\mathrm{~m}, 2 \mathrm{H}), 3.28\left(\mathrm{dd},{ }^{3} J(\mathrm{H}, \mathrm{H})=6.6 \mathrm{~Hz}\right.$, $\left.{ }^{3} J(\mathrm{H}, \mathrm{H})=6.9 \mathrm{~Hz}, 1 \mathrm{H}\right), 3.04\left(\mathrm{dd},{ }^{3} J(\mathrm{H}, \mathrm{H})=5.9 \mathrm{~Hz},{ }^{2} J(\mathrm{H}, \mathrm{H})=15.1 \mathrm{~Hz}, 1 \mathrm{H}\right), 3.01(\mathrm{t}$, $\left.{ }^{3} J(\mathrm{H}, \mathrm{H})=6.9 \mathrm{~Hz}, 2 \mathrm{H}\right), 2.89\left(\mathrm{dd},{ }^{3} J(\mathrm{H}, \mathrm{H})=7.5 \mathrm{~Hz},{ }^{2} J(\mathrm{H}, \mathrm{H})=15.1 \mathrm{~Hz}, 1 \mathrm{H}\right), 1.69-$ $1.32\left(\right.$ several m, 21H), $0.92\left(\mathrm{~d},{ }^{3} J(\mathrm{H}, \mathrm{H})=6.5 \mathrm{~Hz}, 3 \mathrm{H}\right), 0.90\left(\mathrm{~d},{ }^{3} J(\mathrm{H}, \mathrm{H})=6.0 \mathrm{~Hz}, 3 \mathrm{H}\right)$, $0.89\left(\mathrm{~d},{ }^{3} J(\mathrm{H}, \mathrm{H})=6.5 \mathrm{~Hz}, 3 \mathrm{H}\right), 0.85\left(\mathrm{~d},{ }^{3} \mathrm{~J}(\mathrm{H}, \mathrm{H})=6.0 \mathrm{~Hz}, 3 \mathrm{H}\right),{ }^{13} \mathrm{C}$ NMR $(125 \mathrm{MHz}$, $\mathrm{CD}_{3} \mathrm{OD}$ ): $\delta 178.2(\mathrm{~s}), 177.6(\mathrm{~s}), 174.7$ (s), 173.2 (s), 158.5 (s), 143.7 (s), 139.6 (d), 137.7 (s), 130.9 (d), 129.4 (d), 129.3 (d), 121.2 (d), 79.8 (s), 76.9 (s), 56.1 (d), 55.1 (d), $53.5(\mathrm{~d}), 53.1(\mathrm{~d}), 41.9(\mathrm{t}), 41.8(\mathrm{t}), 41.2(\mathrm{t}), 35.9(\mathrm{t}), 30.9(\mathrm{t}), 30.7(\mathrm{t}), 28.8(\mathrm{q})$, 25.9 (d), $25.8(\mathrm{~d}), 24.0(\mathrm{t}), 23.7(\mathrm{q}), 23.5(\mathrm{q}), 22.0(\mathrm{q}), 21.5(\mathrm{q})$; MS (ESI, $\left.\mathrm{CH}_{2} \mathrm{Cl}_{2} / \mathrm{MeOH} 20: 1\right): \quad m / z(\%) 852(100)[\mathrm{M}+\mathrm{H}]^{+}, 873(77)[\mathrm{M}+\mathrm{Na}]^{+}, 1702(81)$ $[2 \mathrm{M}+\mathrm{H}]^{+}, 1725(60)[2 \mathrm{M}+\mathrm{Na}]^{+}$; anal. calc. for $\mathrm{C}_{48} \mathrm{H}_{66} \mathrm{~N}_{8} \mathrm{O}_{6}(1.3 \mathrm{MeOH})$ (892.72): $\mathrm{C}$ 66.32, H 8.04, N 12.55; found C 66.32, H 8.29, N 12.72.

Fmoc-Leu-Lys(Boc)-Leu-His(Trt)-Leu-NH $\mathbf{N H}_{2} \quad$ Coupling of H-Lys(Boc)-LeuHis(Trt)-Leu- $\mathrm{NH}_{2}(655 \mathrm{mg}, 0.77 \mathrm{mmol})$ and Fmoc-Leu-OH (325 mg, $\left.0.92 \mathrm{mmol}\right)$ following procedure $\mathrm{A}$ and purification of the crude product by column chromatography $\left(\mathrm{CH}_{2} \mathrm{Cl}_{2} / \mathrm{MeOH} 20: 1\right.$, then 10:1) yielded pure Fmoc-Leu-Lys(Boc)Leu-His(Trt)-Leu- $\mathrm{NH}_{2}(520 \mathrm{mg}, 57 \%)$ as a colorless powder. TLC $\left(\mathrm{CH}_{2} \mathrm{Cl}_{2} / \mathrm{MeOH}\right.$ 10:1): $R_{\mathrm{f}} 0.42 ;[\alpha]_{\mathrm{D}}^{20}=-16.2\left(c=1.0, \mathrm{CH}_{3} \mathrm{OH}\right) ; \mathrm{mp}=161-162{ }^{\circ} \mathrm{C} ; \mathrm{IR}: \mathrm{v} 3300(\mathrm{~m})$, $2956(\mathrm{~m}), 1652$ (s), 1526 (s), 1447 (w), 1365 (w), 1251 (s), $1166(\mathrm{~m}), 1036(\mathrm{w}), 867$ (w), $739(\mathrm{~m}), 700(\mathrm{~s}) ;{ }^{1} \mathrm{H}$ NMR $\left(400 \mathrm{MHz}, \mathrm{CD}_{3} \mathrm{OD}\right): \delta 7.83\left(\mathrm{~d},{ }^{3} J(\mathrm{H}, \mathrm{H})=6.8 \mathrm{~Hz}\right.$, $1 \mathrm{H}), 7.81\left(\mathrm{~d},{ }^{3} J(\mathrm{H}, \mathrm{H})=6.6 \mathrm{~Hz}, 1 \mathrm{H}\right), 7.68\left(\mathrm{~d},{ }^{3} J(\mathrm{H}, \mathrm{H})=7.6 \mathrm{~Hz}, 1 \mathrm{H}\right), 7.58\left(\mathrm{~d},{ }^{3} J(\mathrm{H}, \mathrm{H})\right.$ $=7.3 \mathrm{~Hz}, 1 \mathrm{H}), 7.43-7.20(\mathrm{~m}, 14 \mathrm{H}), 7.13-6.98(\mathrm{~m}, 6 \mathrm{H}), 6.79(\mathrm{~s}, 1 \mathrm{H}), 4.50(\mathrm{dd}$, $\left.{ }^{3} J(\mathrm{H}, \mathrm{H})=4.3 \mathrm{~Hz},{ }^{3} J(\mathrm{H}, \mathrm{H})=9.6 \mathrm{~Hz}, 1 \mathrm{H}\right), 4.34\left(\mathrm{dd},{ }^{3} J(\mathrm{H}, \mathrm{H})=3.7 \mathrm{~Hz},{ }^{3} J(\mathrm{H}, \mathrm{H})=10.7\right.$ $\mathrm{Hz}, 1 \mathrm{H}), 4.22-4.14(\mathrm{~m}, 3 \mathrm{H}), 4.03\left(\mathrm{t},{ }^{3} J(\mathrm{H}, \mathrm{H})=6.7 \mathrm{~Hz}, 1 \mathrm{H}\right), 3.81\left(\mathrm{dd},{ }^{3} J(\mathrm{H}, \mathrm{H})=7.3\right.$ $\left.\mathrm{Hz},{ }^{3} J(\mathrm{H}, \mathrm{H})=8.3 \mathrm{~Hz}, 1 \mathrm{H}\right), 3.50\left(\mathrm{t},{ }^{3} J(\mathrm{H}, \mathrm{H})=7.6 \mathrm{~Hz}, 1 \mathrm{H}\right), 3.16-2.96(\mathrm{~m}, 4 \mathrm{H}), 1.76-$ $1.28\left(\right.$ several m, 21H), $0.90\left(\mathrm{~d},{ }^{3} J(\mathrm{H}, \mathrm{H})=6.3 \mathrm{~Hz}, 3 \mathrm{H}\right), 0.87\left(\mathrm{~d},{ }^{3} J(\mathrm{H}, \mathrm{H})=5.7 \mathrm{~Hz}, 3 \mathrm{H}\right)$, $0.85\left(\mathrm{~d},{ }^{3} J(\mathrm{H}, \mathrm{H})=5.7 \mathrm{~Hz}, 3 \mathrm{H}\right), 0.83\left(\mathrm{~d},{ }^{3} J(\mathrm{H}, \mathrm{H})=6.3 \mathrm{~Hz}, 3 \mathrm{H}\right), 0.81\left(\mathrm{~d},{ }^{3} J(\mathrm{H}, \mathrm{H})=5.7\right.$ $\mathrm{Hz}, 3 \mathrm{H}), 0.79\left(\mathrm{~d},{ }^{3} \mathrm{~J}(\mathrm{H}, \mathrm{H})=5.7 \mathrm{~Hz}, 3 \mathrm{H}\right) ;{ }^{13} \mathrm{C} \mathrm{NMR}\left(100 \mathrm{MHz}, \mathrm{CD}_{3} \mathrm{OD}\right): \delta 177.9(\mathrm{~s})$, 177.0 (s), 176.0 (s), 175.8 (s), 173.7 (s), 173.7 (s), 159.1 (s), 145.4 (s), 145.1 (s), 143.8 (s), 142.9 (s), 142.8 (s), 139.4 (d), 138.1 (s), 131.1 (d), 131.0 (d), 130.9 (d), 129.4 (d), 129.3 (d), 129.1 (d), 128.3 (d), 126.5 (d), 126.3 (d), 121.3 (d), 121.2 (d), $80.0(\mathrm{~s}), 76.9$ (s), 68.8 (t), $56.8(\mathrm{~d}), 56.6(\mathrm{~d}), 56.1(\mathrm{~d}), 54.7$ (d), $53.4(\mathrm{~d}), 48.0(\mathrm{~d}), 41.6$ $(\mathrm{t}), 41.2(\mathrm{t}), 40.6(\mathrm{t}), 31.7(\mathrm{t}), 31.1(\mathrm{t}), 30.7(\mathrm{t}), 28.9(\mathrm{q}), 26.8(\mathrm{~d}), 25.9(\mathrm{~d}), 25.9(\mathrm{~d})$, 
$25.7(\mathrm{t}), 24.0(\mathrm{q}), 23.9(\mathrm{q}), 23.0(\mathrm{q}), 22.9$ (q), $21.4(\mathrm{q}), 21.3$ (q); MS (ESI, $\left.\mathrm{CH}_{2} \mathrm{Cl}_{2} / \mathrm{MeOH} 10: 1\right): m / z(\%) 1208.7$ (100) [M+Na].

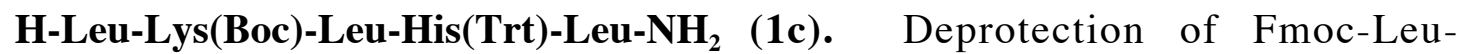
Lys(Boc)-Leu-His(Trt)-Leu- $\mathrm{NH}_{2}(520 \mathrm{mg}, 0.44 \mathrm{mmol})$ following procedure $\mathrm{B}$ and purification of the crude product by column chromatography $\left(\mathrm{CH}_{2} \mathrm{Cl}_{2} / \mathrm{MeOH} 10: 1\right)$ yielded pure 1c (385 mg, 91\%) as a colorless powder. TLC $\left(\mathrm{CH}_{2} \mathrm{Cl}_{2} / \mathrm{MeOH} 10: 1\right): R_{\mathrm{f}}$ 0.05; HPLC (YMC-Pack SIL, $250 \times 10 \mathrm{~mm}, \mathrm{CH}_{2} \mathrm{Cl}_{2} / \mathrm{MeOH} / \mathrm{Et}_{3} \mathrm{~N}$ 94.9:5:0.1, 2 $\left.\mathrm{ml} / \mathrm{min}, t_{\mathrm{R}}=8.9 \mathrm{~min}\right) ;[\alpha]^{20}{ }_{\mathrm{D}}=-15.7\left(c=0.1, \mathrm{CH}_{3} \mathrm{OH}\right) ; \mathrm{mp}=159-160{ }^{\circ} \mathrm{C} ; \mathrm{IR}: \quad v$ $3286(\mathrm{~m}), 2955(\mathrm{~m}), 1629$ (s), 1520 (s), $1446(\mathrm{w}), 1365$ (w), $1248(\mathrm{~m}), 1168(\mathrm{~s}), 1001$ (w), $870(\mathrm{w}), 748(\mathrm{~m}), 700(\mathrm{~s}) ;{ }^{1} \mathrm{H}$ NMR $\left(400 \mathrm{MHz}, \mathrm{CD}_{3} \mathrm{OD}\right): \delta 7.41-7.36(\mathrm{~m}, 10 \mathrm{H})$, $7.14-7.12(\mathrm{~m}, 6 \mathrm{H}), 6.78(\mathrm{~s}, 1 \mathrm{H}), 4.55\left(\mathrm{dd},{ }^{3} J(\mathrm{H}, \mathrm{H})=6.1 \mathrm{~Hz},{ }^{3} J(\mathrm{H}, \mathrm{H})=7.6 \mathrm{~Hz}\right), 4.37$ $-4.25(\mathrm{~m}, 3 \mathrm{H}), 3.38\left(\mathrm{dd},{ }^{3} J(\mathrm{H}, \mathrm{H})=6.7 \mathrm{~Hz},{ }^{3} J(\mathrm{H}, \mathrm{H})=8.7 \mathrm{~Hz}, 1 \mathrm{H}\right), 3.08\left(\mathrm{dd},{ }^{3} J(\mathrm{H}, \mathrm{H})=\right.$ $\left.6.1 \mathrm{~Hz},{ }^{2} J(\mathrm{H}, \mathrm{H})=15.0 \mathrm{~Hz}, 1 \mathrm{H}\right), 3.01\left(\mathrm{t},{ }^{3} J(\mathrm{H}, \mathrm{H})=6.6 \mathrm{~Hz}, 2 \mathrm{H}\right), 2.90\left(\mathrm{dd},{ }^{3} J(\mathrm{H}, \mathrm{H})=\right.$ $\left.7.6 \mathrm{~Hz},{ }^{2} \mathrm{~J}(\mathrm{H}, \mathrm{H})=15.0 \mathrm{~Hz}, 1 \mathrm{H}\right), 1.83-1.26($ several m, $24 \mathrm{H}), 0.94\left(\mathrm{~d},{ }^{3} \mathrm{~J}(\mathrm{H}, \mathrm{H})=6.4\right.$ $\mathrm{Hz}, 3 \mathrm{H}), 0.92\left(\mathrm{~d},{ }^{3} J(\mathrm{H}, \mathrm{H})=5.2 \mathrm{~Hz}, 3 \mathrm{H}\right), 0.91\left(\mathrm{~d},{ }^{3} J(\mathrm{H}, \mathrm{H})=6.3 \mathrm{~Hz}, 3 \mathrm{H}\right), 0.90(\mathrm{~d}$, $\left.{ }^{3} J(\mathrm{H}, \mathrm{H})=6.4 \mathrm{~Hz}, 3 \mathrm{H}\right), 0.88\left(\mathrm{~d},{ }^{3} J(\mathrm{H}, \mathrm{H})=6.3 \mathrm{~Hz}, 3 \mathrm{H}\right), 0.86\left(\mathrm{~d},{ }^{3} J(\mathrm{H}, \mathrm{H})=5.2 \mathrm{~Hz}, 3 \mathrm{H}\right)$; ${ }^{13} \mathrm{C}$ NMR (100 MHz, CD $\left.\mathrm{CD}_{3} \mathrm{OD}\right): \delta 178.2(\mathrm{~s}), 177.6(\mathrm{~s}), 174.6$ (s), 174.5 (s), 173.2 (s), 158.5 (s), 143.7 (s), 139.6 (d), 137.6 (s), 130.9 (d), 129.4 (d), 129.3 (d), 121.1 (d), $79.8(\mathrm{~s}), 76.9(\mathrm{~s}), 55.2(\mathrm{~d}), 54.8(\mathrm{~d}), 54.5(\mathrm{~d}), 53.6(\mathrm{~d}), 53.1(\mathrm{~d}), 45.6(\mathrm{t}), 41.8(\mathrm{t}), 41.1$ $(\mathrm{t}), 32.6(\mathrm{t}), 31.1(\mathrm{t}), 30.6(\mathrm{t}), 28.9(\mathrm{q}), 25.8(\mathrm{~d}), 24.2(\mathrm{t}), 23.7(\mathrm{q}), 23.6(\mathrm{q}), 23.5(\mathrm{q})$, 22.5 (q), 21.9 (q), 21.7 (q); MS (ESI, $\mathrm{CH}_{2} \mathrm{Cl}_{2} / \mathrm{MeOH}$ 9:1): $\mathrm{m} / z$ (\%) 965 (100) $[\mathrm{M}+\mathrm{H}]^{+}$, $987(73)[\mathrm{M}+\mathrm{Na}]^{+}, 1952(37)[2 \mathrm{M}+\mathrm{Na}]^{+}$; anal. calc. for $\mathrm{C}_{54} \mathrm{H}_{77} \mathrm{~N}_{9} \mathrm{O}_{7}(0.5 \mathrm{MeOH})$ (980.27): C 66.77, H 8.12, N 12.86; found C 66.77, H 8.25, N 13.00.

\section{$1^{3}, 2^{3}, 3^{2}, 4^{3}, 5^{2}, 6^{3}, 7^{2}, 8^{3}$-Octakis(Gla-Leu-Lys(Boc)-Leu-His(Trt)-Leu-NH ${ }_{2}$ )- $p$ -}

octiphenyl 1d. To a solution of $p$-octiphenyl 1a $(11 \mathrm{mg}, 9.14 \mu \mathrm{mol})$ in dried DMF $(0.8 \mathrm{ml}), \mathbf{1 c}(92 \mathrm{mg}, 95 \mu \mathrm{mol}), \mathrm{HATU}(47 \mathrm{mg}, 124 \mu \mathrm{mol})$, and dried triethylamine (85 $\mu 1,585 \mu \mathrm{mol})$ were added. After stirring for $80 \mathrm{~min}$ at $\mathrm{rt}$, the reaction mixture was concentrated in vacuo. Purification of the crude product by Sephadex LH-20 column $(\mathrm{MeOH})$ yielded pure 1d $(17.8 \mathrm{mg}, 22 \%)$ as a colorless solid. HPLC (YMC-Pack CN, $\left.50 \times 4 \mathrm{~mm}, \mathrm{CH}_{2} \mathrm{Cl}_{2} /\left(\mathrm{MeOH}+1 \% \mathrm{Et}_{3} \mathrm{~N}\right) 95: 5,1 \mathrm{ml} / \mathrm{min}, t_{\mathrm{R}}=0.36 \mathrm{~min}\right) ;{ }^{1} \mathrm{H} \mathrm{NMR}(300$ $\left.\mathrm{MHz}, \mathrm{CD}_{3} \mathrm{OD}\right): \delta 7.55-7.30(\mathrm{~m}, 104 \mathrm{H}), 7.13-7.09(\mathrm{~m}, 48 \mathrm{H}), 7.01(\mathrm{~m}, 2 \mathrm{H}), 6.80-$ $6.76(\mathrm{~m}, 8 \mathrm{H}), 4.59-4.37$ (several $\mathrm{m}, 56 \mathrm{H}), 3.05-2.96(\mathrm{~m}, 32 \mathrm{H}), 1.64$ - 1.17 (several $\mathrm{m}, 192 \mathrm{H}), 0.93-0.72$ (m, 144H); MS (ESI, $\left.\mathrm{CH}_{3} \mathrm{CN} / \mathrm{H}_{2} \mathrm{O} / \mathrm{AcOH} 74: 24: 2\right): \mathrm{m} / \mathrm{z}(\%)$ $1097(60)[\mathrm{M}+\mathrm{H}]^{8+}, 1254(100)[\mathrm{M}+\mathrm{H}]^{7+}, 1270(100)[\mathrm{M}+2 \mathrm{AcOH}]^{7+}, 1463$ (60) $[\mathrm{M}+\mathrm{H}]^{6+}, 1482(50)[\mathrm{M}+2 \mathrm{AcOH}]^{6+}, 1756(30)[\mathrm{M}+\mathrm{H}]^{5+}$.

$1^{3}, 2^{3}, 3^{2}, 4^{3}, 5^{2}, 6^{3}, 7^{2}, 8^{3}$-Octakis(Gla-Leu-Lys-Leu-His-Leu-NH $)$-p-octiphenyl $1^{m}$ (=

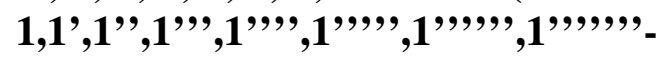
\{[1,1:4',1":4",1",:4",,1",":4",",1",",:4,",",1,",",:4,",",,1",",",-octiphenyl]-

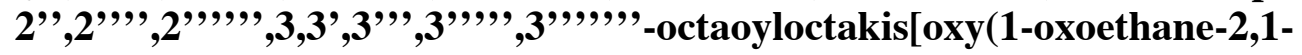

diyl)]\}octakis[L-leucyl-L-lysyl-L-leucyl-L-histidyl-L-leucinamide]. Compound 1d (17.8 mg, $2.03 \mu \mathrm{mol})$ was dissolved in TFA (4 ml). After stirring for $30 \mathrm{~min}$ at $\mathrm{rt}$, the reaction mixture was concentrated in vacuo. Nonpolar impurities were removed by solid-liquid extraction ( 3 times with hexane). Purification by RP HPLC (YMC-Pack ODS-A, $\left.250 \times 10 \mathrm{~mm}, \mathrm{MeOH} / \mathrm{H}_{2} \mathrm{O} / \mathrm{TFA} 94: 5: 1,2 \mathrm{ml} / \mathrm{min}, t_{\mathrm{R}}=4.76 \mathrm{~min}\right)$ yielded 
pure $\mathbf{1}^{m}$ (12 mg, quant) as a colorless TFA/phosphate salt (see ESI-MS). ${ }^{1} \mathrm{H}$ NMR (300 MHz, $\left.\mathrm{CD}_{3} \mathrm{OD}\right): \delta 8.77$ - $8.75(\mathrm{~m}, 4 \mathrm{H}), 8.42$ - $8.20(\mathrm{~m}, 8 \mathrm{H}), 7.52$ - $7.24(\mathrm{~m}, 32 \mathrm{H})$, $7.06-7.04(\mathrm{~m}, 2 \mathrm{H}), 6.80-6.76(\mathrm{~m}, 8 \mathrm{H}), 4.74-4.72(\mathrm{~m}, 24 \mathrm{H}), 4.41-4.38(\mathrm{~m}, 32 \mathrm{H})$, $3.30-2.92(\mathrm{~m}, 32 \mathrm{H}), 2.00-1.30$ (several $\mathrm{m}, 120 \mathrm{H}), 0.93-0.72(\mathrm{~m}, 144 \mathrm{H})$; MS (ESI, $\left.\mathrm{CH}_{3} \mathrm{CN} / \mathrm{H}_{2} \mathrm{O} / \mathrm{AcOH} 74: 24: 2\right): \mathrm{m} / z(\%) 755(35)[\mathrm{M}+\mathrm{H}]^{8+}, 769$ (44) $[\mathrm{M}+\mathrm{TFA}]^{8+}, 863$ $(100)[\mathrm{M}+\mathrm{H}]^{7+}, 877(90)\left[\mathrm{M}+\mathrm{H}_{3} \mathrm{PO}_{4}\right]^{7+}, 879(70)[\mathrm{M}+\mathrm{TFA}]^{7+}, 891(60)\left[\mathrm{M}+2 \mathrm{H}_{3} \mathrm{PO}_{4}\right]^{7+}$, $893(65)\left[\mathrm{M}+\mathrm{H}_{3} \mathrm{PO}_{4}+\mathrm{TFA}\right]^{7+}, 1007(55)[\mathrm{M}+\mathrm{H}]^{6+}, 1023(45)\left[\mathrm{M}+\mathrm{H}_{3} \mathrm{PO}_{4}\right]^{6+}, 1042$ (45) $\left[\mathrm{M}+\mathrm{H}_{3} \mathrm{PO}_{4}+\mathrm{TFA}\right]^{6+}, 1098(45)\left[\mathrm{M}+\mathrm{H}_{3} \mathrm{PO}_{4}+4 \mathrm{TFA}\right]^{6+}, 1207$ (10) $[\mathrm{M}+\mathrm{H}]^{5+}$ (compare Tab. 1). Stock solutions for studies on activity were prepared in methanol, monomer concentrations were corroborated by UV-visible $\left[\varepsilon(p\right.$-octiphenyl $)=28.6 \mathrm{mM}^{-1} \cdot \mathrm{cm}^{-1}$ $(320 \mathrm{~nm})$ in water]. ${ }^{\mathrm{S}}$

$1^{3}, 2^{3}, 3^{2}, 4^{3}, 5^{2}, 6^{3}, 7^{2}, 8^{3}$-Octakis(Gla-Leu-His-Leu-His-Leu-NH $)$ - $p$-octiphenyl $\quad\left(2^{m}\right)$ and $1^{3}, 2^{3}, 3^{2}, 4^{3}, 5^{2}, 6^{3}, 7^{2}, 8^{3}$-Octakis(Gla-Leu-Arg-Leu-His-Leu-NH - $_{2}$-p-octiphenyl $\left(3^{m}\right)$. These compounds were prepared following previously reported procedures. ${ }^{\mathrm{S} 1, \mathrm{S3}}$

EYPC-LUVs $\supset$ ANTS/DPX. Stock solutions of large unilamellar vesicles composed of egg yolk phosphatidylcholine and loaded with ANTS and DPX were prepared by freeze-thaw-extrusion following the previously described method without change. ${ }^{\mathrm{S}}$ Final conditions: $\sim 5 \mathrm{mM}$ EYPC; inside: $12.5 \mathrm{mM}$ ANTS, $45.0 \mathrm{mM}$ DPX, $5 \mathrm{mM}$ TES, $20 \mathrm{mM} \mathrm{KCl}$, pH 7.0; outside: $5 \mathrm{mM}$ TES, $100 \mathrm{mM} \mathrm{KCl}, \mathrm{pH}=7.0$.

ANTS/DPX assay. General procedure: EYPC-LUVs $\supset$ ANTS/DPX $(100 \mu 1)$ were added to gently stirred, thermostated buffer $(1.90 \mathrm{ml}, 10 \mathrm{mM}$ MES, $100 \mathrm{mM} \mathrm{KCl}, \mathrm{pH}$ $=4.5 \sim 7.0)$ in a fluorescence cuvette. Fluorescence emission intensity $I_{\mathrm{t}}\left(\lambda_{\mathrm{em}}=520\right.$ $\left.\mathrm{nm}, \lambda_{\mathrm{ex}}=353 \mathrm{~nm}\right)$ was monitored as a function of time $(t)$ during addition of $20 \mu 1 \mathbf{1}^{\mathbf{m}}$ $(0-200 \mu \mathrm{M}$, final concentration $0-2 \mu \mathrm{M})$ and, after $5 \mathrm{~min}, 40 \mu 11.2 \%$ aq triton $\mathrm{X}-$ 100. Fluorescence kinetics were normalized to fractional emission intensity $I^{\mathrm{n}}$ using equation [S1]

$$
I^{\mathrm{n}}=\left(I_{\mathrm{t}}-I_{0}\right) /\left(I_{\infty}-I_{0}\right)
$$

$[\mathrm{S} 1]$,

where $I_{0}=I_{\mathrm{t}}$ at pore addition, $I_{\infty}=I_{\mathrm{t}}$ at saturation after lysis. Obtained $I^{\mathrm{n}}$ at time $t\left(I_{\mathrm{t}}^{\mathrm{n}}\right)$ was further converted into fractional pore activity $Y$ using equation [S2]

$$
Y=\left[\left(I_{\mathrm{t}}^{\mathrm{n}}-I_{\mathrm{MIN}}^{\mathrm{n}}\right) /\left(I_{\mathrm{MAX}}^{\mathrm{n}}-I_{\mathrm{MIN}}^{\mathrm{n}}\right)\right]
$$

where $I^{\mathrm{n}}{ }_{\text {MIN }}$ is $I^{\mathrm{n}}$ tobtained under the conditions giving the lowest activity, and $I_{\text {MAX }}^{\mathrm{n}}$ is the same for the highest activity.

Pore Blockage in EYPC-LUVs $\supset$ ANTS/DPX. Stock solutions of PGA and ATP were prepared in buffer ( $10 \mathrm{mM}$ MES, $100 \mathrm{mM} \mathrm{KCl}, \mathrm{pH} 5.0)$ and $\mathrm{pH}$ was adjusted. ${ }^{\mathrm{S}}$ $20 \mu \mathrm{L}$ of blocker stock solution was taken and added to $2 \mathrm{~mL}$ gently stirred EYPCLUVs $\supset$ ANTS/DPX suspension (pH 5.0) in a thermostated fluorescence cuvette prepared as described in above general procedure (final concentrations ATP: $100 \mu \mathrm{M}$ - $10 \mathrm{mM}$, PGA: $5 \mathrm{nM}-5 \mu \mathrm{M})$. Then, pore 1 was added $(30 \mu \mathrm{l}$ of $80 \mu \mathrm{M}$ monomer $\mathbf{1}^{\mathbf{m}}$ in $\mathrm{MeOH}, 1.2 \mu \mathrm{M}$ final monomer concentration) and fractional activity of pore $Y$ 
was determined as a function of blocker concentration as described in above general procedure. Dissociation constants $K_{\mathrm{D}}$ and Hill coefficients $n$ were determined by curve fitting of the obtained dose response curves to the Hill equation [S3]

$$
Y=Y_{\infty}+\left(Y_{0}-Y_{\infty}\right) /\left\{1+\left([\text { blocker }] / K_{\mathrm{D}}\right)^{n}\right\}
$$

where $Y_{0}$ is $Y$ without blocker, and $Y_{\infty}$ is $Y$ with excess blocker.

Planar Bilayer Conductance Measurements. General procedures have been described in refs. S1 and S6. In brief, $n$-decane containing EYPC $(33 \mathrm{mg} / \mathrm{ml})$ was painted on an orifice $(\mathrm{d}=150 \mu \mathrm{m})$ separating the two chambers, and $\mathbf{1}^{m}(4 \mu \mathrm{M}$ final $)$ was added to cis (trans at ground). Currents were recorded as a function of time and analyzed following the protocol described (Conditions: $2.0 \mathrm{M} \mathrm{KCl}$, symmetric, agar bridge: $2 \mathrm{M} \mathrm{KCl}, 2 \%$ agar; electrodes: $\mathrm{Ag} / \mathrm{AgCl}$; Bessel filter: $1 \mathrm{kHz}$; sampled at 10 $\mathrm{kHz}$ ). The $I-V$ curve was obtained by plotting the single channel current as a function of membrane potential $V$, and analyzed according to Ohm's law. The pore conductance obtained from the slope of the $I-V$ curve was converted into the diameter $d$ using the Hille equation $[\mathrm{S} 4]^{\mathrm{S} 6, \mathrm{~S} 7}$

$$
1 / g=l \rho /\left[\pi(d / 2)^{2}\right]+\rho / d
$$

where $l$ is the ion channel length ( $34 \AA$ ) and $\rho$ the resistivity of the recording solution $\left[\rho=5.21 \Omega \mathrm{cm}^{\mathrm{S} 8}\right]$. In pore blockage experiments, HPTS was added to the trans chamber at indicated concentrations and changes of the macroscopic current $I$ were recorded as a function of the concentration of HPTS. (Conditions as above except: 5

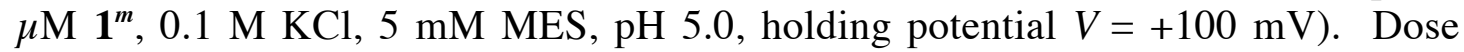
response curves were fitted to the Hill equation [S3].

Esterolysis. $1980 \mu \mathrm{l}$ buffer (10 mM MES, $100 \mathrm{mM} \mathrm{KCl,} \mathrm{pH} 4.0 \sim 7.0)$ were added into a fluorescence cell equipped with a magnetic stirr bar and placed in a thermostatted cell holder $\left(25^{\circ} \mathrm{C}\right)$. Then, $20-50 \mu 1$ of freshly prepared aqueous stock solutions of AcTPS 5 were added to give $0-2 \mu \mathrm{M}$ final substrate concentration. After addition of $20 \mu 1$ of $\mathbf{1}^{\boldsymbol{m}}$ (in $\mathrm{MeOH}$ ), $\mathbf{2}^{\boldsymbol{m}}$ or $\mathbf{3}^{\boldsymbol{m}}$ (in DMSO, final concentrations 0 $5 \mu \mathrm{M})$, changes in HPTS emission $\left(\lambda_{\mathrm{em}}=510 \mathrm{~nm}, \lambda_{\mathrm{ex}}=415.5 \mathrm{~nm}\right)$ were recorded as a function of time. Initial velocities of HPTS formation were obtained by comparison with calibration curves. The obtained $c_{\mathrm{S}}$ and $c_{\mathrm{M}}$ curves were analyzed using the Michaelis-Menten and the Hill equation [S3], respectively.

S1) Baumeister, B.; Sakai, N.; Matile, S. Org. Lett. 2001, 3, 4229-4232.

S2) Weiss, L. A.; Sakai, N.; Ghebremariam, B.; Ni, C.; Matile, S. J. Am. Chem. Soc. 1997, 119, 12142-12149.

S3) Sordé, N.; Matile, S. J. Supramol. Chem. 2002, 2, 191-199.

S4) Talukdar, P.; Sakai, N.; Sordé, N.; Gerard, D.; Cardona, V. M. F.; Matile, S. Bioorg. Med. Chem. 2004, 12, 1325-1336.

S5) Das, G.; Onouchi, H.; Yashima, E.; Sakai, N.; Matile, S. ChemBioChem 2002, 3, 1089-1096. 
S6) Som, A.; Sakai, N.; Matile, S. Bioorg. Med. Chem. 2003, 11, 1363-1369.

S7) Hille, B. Ionic Channels of Excitable Membranes, 2nd ed.; Sinauer Associates, Inc.: Sunderland, MA, 1992.

S8) Sakai, N.; Ni, C.; Bezrukov, S. M.; Matile, S. Bioorg. Med. Chem. Lett. 1998, 8, 2743-2747. 